\title{
Comparing EKF and SPKF Algorithms for Simultaneous Localization and Mapping (SLAM)
}

\author{
Zolghadr Javad, Yuanli Cai*, Yekkehfallah Majid \\ School of electronic and information engineering, Xi'an Jiaotong University, Xi'an 710049, China \\ E-mail addresses: Javad.zolghadr@gmail.com,ylicai@mail.xjtu.edu.cn, Majid.fallah@stu.xjtu.edu.cn,
}

\begin{abstract}
Simultaneous Localization and Mapping (SLAM) is the problem in which a sensor-enabled mobile robot incrementally builds a map for an unknown environment, while localizing itself within this map. A problem with detection of correct path of moving objects is the received noisy data. Therefore, it is possible that the information is incorrectly detected. The Kalman Filter's linearized error propagation can result in big errors and instability in the SLAM problem. One approach to reduce this situation is using of iteration in Extended Kalman Filter (EKF) and Sigma Point Kalman Filter (SPKF). We will show that the recapitulate versions of kalman filters can improve the estimation accuracy and robustness of these filters beside of linear error propagation. Simulation results are presented to validate this improvement of state estimate convergence through repetitive linearization of the nonlinear model in EKF and SPKF for SLAM algorithms. Results of this evaluation are introduced by computer simulations and verified by offline implementation of the SLAM algorithm on mobile robot in MRL Robotic Lab.
\end{abstract}

Keywords: Extended Kalman Filter, Sigma Point Kalman Filter, SLAM, instability, Mobile Robot, Nonlinear Estimation.

\section{Introduction}

Simulation Localization and Mapping (SLAM) has the problem of incrementally building a spatial consistent map from noisy sensor data gathered by a robot and tracking robot pose with the built map [1], [2], [11]. The Kalman filter is a very general method of filtering which can solve problems such as optimal estimation, prediction, noise filtering, and optimal control. In the past decade, the SLAM related research has received an increasing and extensive attention in theory and application level. Due to the mutual dependence of robot pose and the map, the noise of robot pose arise the uncertainty of map estimation and vice versa. Therefore, this problem requires a solution in a high dimensional space.

Numerous method have been used to address the SLAM problem, an overview of which is presented in [3], [4]. One of the most popular approaches to the SLAM problems are the extended Kalman filter (EKF-SLAM).
The effectiveness of the EKF approach lies on the fact that it holds a fully correlated posterior over feature maps and vehicle poses [3], [12]. Due to the inherent non-linearity of the SLAM problem, it applies the Kalman filter framework to nonlinear Gaussian systems, by employing the first-order Taylor expansion to approximate the non-liner models. However, this approximation treatment often introduces large errors in the estimate of the states and can lead filter to divergence [5]. These serious drawbacks have been confirmed in [4], [6], [7] with carefully designated experiments. Another serious potential drawback of EKF-SLAM is the derivation of the Jacobin matrices, which is really a bothersome process.

Some researchers applied these new extensions of the Kalman filters family called Sigma Point Kalman Filters (SPKF) to solve the SLAM problem [8], [9], [10]. In spite of superiority of the SPKF over the EKF, We will study the future of SPKF over EKF.

\footnotetext{
* Corresponding author.
} 
In this paper, we want to investigate SLAM with SPKF and show this method robustness. The rest of the paper is structured as follows. Section II summarizes the EKF based SLAM. We will briefly describe the SPKF based SLAM in section III. In section, IV the formulation of the EKF and SPKF approaches with their iterated versions in section V. Section VI concludes the paper.

\section{EKF solution for the SLAM}

In this section we summarize the basic EKF SLAM equations to point out the steps in which linearized approximations are introduced. We subsequently analyze their influence in the consistency of the solution obtained. In the standard EKF-based approach to SLAM.
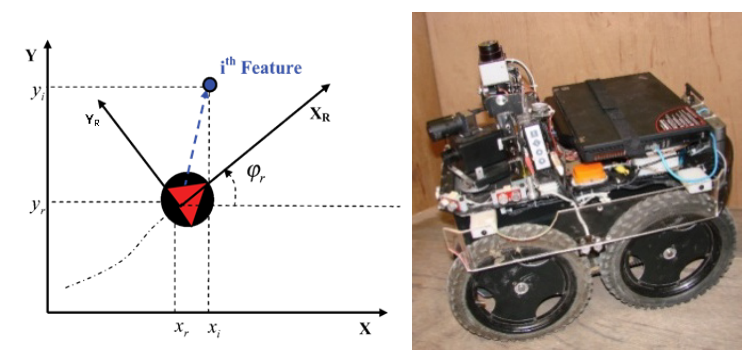

Fig.1. Robot Position and MRL robot 's photos

The Extended Kalman Filter estimates the mean and covariance of the posterior probability distribution function (PDF) of the random state variable $\mathrm{X}$. Let $\wedge$

$X^{+}(k)$ denote the mean of the posterior PDF at time $\mathrm{k}$. The corresponding covariance matrix of the posterior distribution is $B^{+}(k)$. The process model for vehicle and features can be written as:

$$
\left[\begin{array}{l}
X_{v}(k+1) \\
X_{m}(k+1)
\end{array}\right]=\left[\begin{array}{l}
f_{v}\left(X_{v}(k), u(\mathrm{k}+1)\right) \\
\mathrm{X}_{m}(k)
\end{array}\right]+\left[\begin{array}{l}
v(k+1) \\
0
\end{array}\right]
$$

Where $f_{v}$ is the motion model of the Robot, $\mathrm{u}(\mathrm{k})$ is control input and $\mathrm{v} \mathrm{k}(\mathrm{)})$ represents process noise that is zero mean white with covariance $\mathrm{Q}(\mathrm{k})$. The EKF fuses the odomtery measurements with a sequence of observations from the external sensors with the following observation model:

$$
z(k)=h(X(k))+w(k)
$$

$\mathrm{w}(\mathrm{k})$ is zero mean white observation noise with the covariance matrix R (k). Four fundamental stages of the EKF based SLAM are briefly written as follows:

$$
\begin{aligned}
& \text { I. } \quad \text { Prediction } \\
& X^{-}(k+1)=f\left(X^{+}(k), u(k+1)\right), \\
& \hat{z}^{-}(k+1)=\mathrm{h}\left(X^{-}(k+1)\right), \\
& B^{-}(k+1)=\nabla_{x} f(k) \cdot B^{+}(k) \cdot \nabla_{x} f^{T}(k)+Q(k) . \\
& \text { II. } \quad \text { Observation } \\
& v(k+1)=z_{i}(k+1)-\hat{z}^{-}(k+1)
\end{aligned}
$$

Moreover, corresponding matrix:

$S(k+1)=\nabla_{x} h \cdot B^{-}(k+1) \cdot \nabla_{x} h^{T}+R(k+1)$

\section{Update}

Poster distribution's covariance can be same as follows:

$$
\begin{aligned}
& X^{+}(k+1)=X^{-}(k+1)+W(k+1) \cdot \mathrm{v}(k+1) \\
& \mathrm{B}^{+}(k+1)=\mathrm{B}^{-}(k+1)-W \cdot S(k+1) \cdot \mathrm{W}^{T}
\end{aligned}
$$

Kalman gain:

$$
\mathrm{W}(k+1)=\mathrm{B}^{-}(k+1) \cdot \nabla_{X} \mathrm{~h}^{T} \cdot \mathrm{S}^{-1}(k+1)
$$

\section{Consolidation}

After new feature is calculated, estimation must show at last part of consoled state vector:

$X_{i}{ }^{+}(k+1)=g_{i}\left(X_{V}{ }^{-}(k), z(\mathrm{k})\right)$

$g_{i}=$ convert function for polar observation $\mathrm{z}(\mathrm{k})$ to a global Cartesian feature location.

\section{SPKF solution for SLAM}

Mathematic framework of SPKF Based Slam will discuss in this section, in section 2 in this paper assumptions made for SLAM problem, we define a new consolidation state vector that include the original state vector $\mathrm{X}(\mathrm{k})$ and process noise. Block diagonal matrix of $\mathrm{B}(\mathrm{k})$ and process noise covariance matrix $\mathrm{Q}(\mathrm{k})$ is new covariance matrix:

$$
\begin{aligned}
& x_{k}^{a}=\left[X_{k} V_{k}\right]^{T} \\
& B_{k}^{a}=\left[\begin{array}{cc}
B_{k} & 0 \\
0 & O_{k}
\end{array}\right]
\end{aligned}
$$

Sigma point at the time $\mathrm{k}$ will be: 


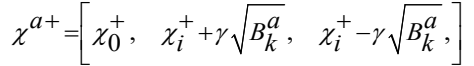

$\gamma$ is a scaling parameter that controls the spread of sigma point around the mean. We can use cholesky factorization for calculate more efficient the covariance matrix square root. These sigma points pass through the process model and transformed sigma points will be calculated:

$$
\chi_{i}^{x-}(k+1)=f\left(\chi_{i}^{x+}(k), u(k), \chi_{i}^{\nu+}(k)\right)
$$

For the original consolidation state vector the $\chi_{i}^{x+}(k)$ is sigma points set and $\chi_{i}^{v+}(k)$ is sigma point set for process noise. The associated covariance matrix and predicted state estimate can be computed as follows:

$\chi_{k+1}^{-}=\sum_{i=0}^{2 N} w_{i}^{m} \chi_{i, k+1}^{x-}$

$B_{k+1}^{-}=\sum_{i=0}^{2 N} \sum_{j=0}^{2 N} w_{i j}^{c}\left(\chi_{i, k+1}^{x-}\right)\left(\chi_{\mathrm{j}, k+1}^{x-}\right)^{T}$

The transformed sigma points pass through the measurement model and samples of predicted are:

$z_{i}^{-}(k+1)=h\left(\chi_{i}^{x-}(k+1)\right)$

Corresponding covariance matrices and predicted measurement can obtain:

$Z_{k+1}^{-}=\sum_{i=0}^{2 N} w_{i}^{m} Z_{i, k+1}^{-}$

Cross covariance matrix between state and measurement $B_{Z Z}^{-}$and covariance matrix and measurement error with assuming correct data are:

$$
\begin{aligned}
& B^{+}(k+1)= \\
& \sum_{i=0}^{2 N} w_{i}\left\{\chi_{i}^{A U G+}(k+1)-\hat{X}^{+}(k+1)\right\}\left\{\chi_{i}^{A U G+}(k+1)-\hat{X}^{+}(k+1)\right\}^{T} \\
& v_{k+1}=z_{k+1}-\hat{z}_{i, k+1} \\
& B_{Z Z, k+1}=R+\sum_{i=0}^{2 N} \sum_{j=0}^{2 N} w_{i j}^{c}\left(z_{i, k+1}^{-}\right)\left(\bar{z}_{\mathrm{j}, k+1}^{-}\right)^{T}
\end{aligned}
$$

With observation noise additively the update state estimate and corresponding covariance matrix can be computed:

$$
\begin{aligned}
& X_{k+1}^{+}=X_{k+1}^{+}+K_{k+1} \cdot v_{k+1}, \\
& B_{k+1}^{+}=B_{k+1}^{-}-K_{k+1} \cdot \bar{B}_{Z Z \cdot k+1}^{-} \cdot K_{k+1}^{T}
\end{aligned}
$$

Kalman gain will be:

$$
K_{k+1}=B_{\bar{X} Z \cdot k+1} \cdot\left[\mathrm{B}_{Z Z \cdot k+1}^{-}\right]^{-1}
$$

After $Z_{k+1}$ detected the consolidation process easily performed with the following equations:

$$
\begin{aligned}
& P_{k+1}^{A U G}=\left[\begin{array}{cc}
P_{k+1}^{-} & 0 \\
0 & R_{k+1}
\end{array}\right] \\
& X_{k+1}^{A U G+}=g_{i}\left(X_{i, k+1}^{A U G}\right) \\
& X k_{k+1}^{+}=\sum_{i=0}^{2 N} w_{i}^{m} \chi_{i, k+1}^{A G U+}
\end{aligned}
$$

In above equations, the parameters $W_{i}^{m}$ and $W_{i j}^{\mathcal{C}}$ are scalar positive valued weights.

In following, we illustrated our results which we implemented on MATLAB:

Table 1. Comparing the Mean Square errors for EKF and SPKF

\begin{tabular}{llll}
\hline $\begin{array}{l}\text { Mean Square Error } \\
\text { (MSE) }\end{array}$ & $x_{R}$ state & $y_{R}$ state & $\varphi_{R}$ state \\
\hline EKF-SLAM & $0 / 622$ & $0 / 1180$ & $0 / 002$ \\
& $0 / 0077$ & $0 / 0120$ & $0 / 0003$
\end{tabular}
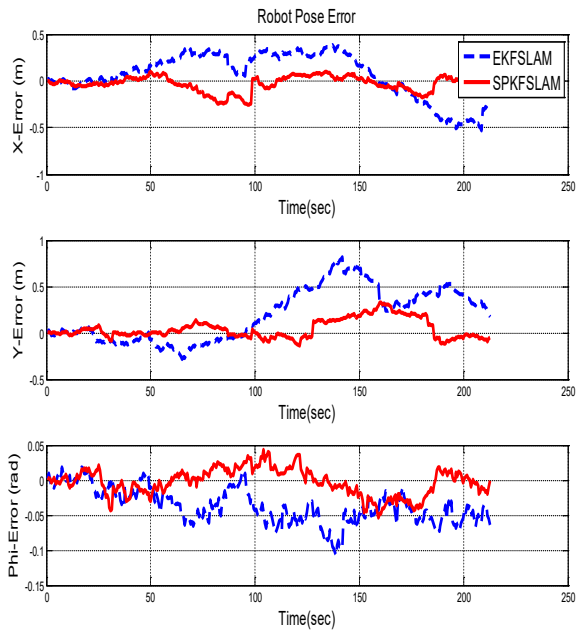

Fig.2. Comparison of vehicle pose estimate errors for EKF (Blue line) vs. SPKF (Red Line) 


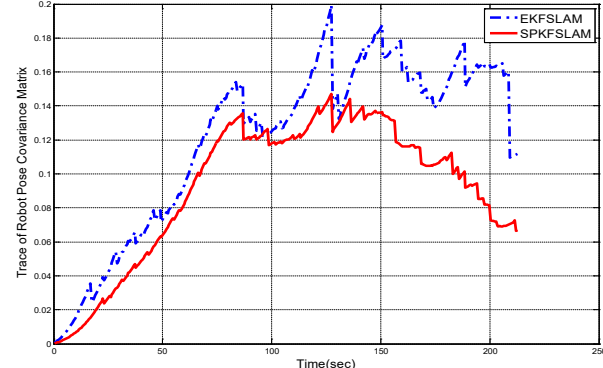

Fig. 3. State Covariance of Robot for KLF and SPKF

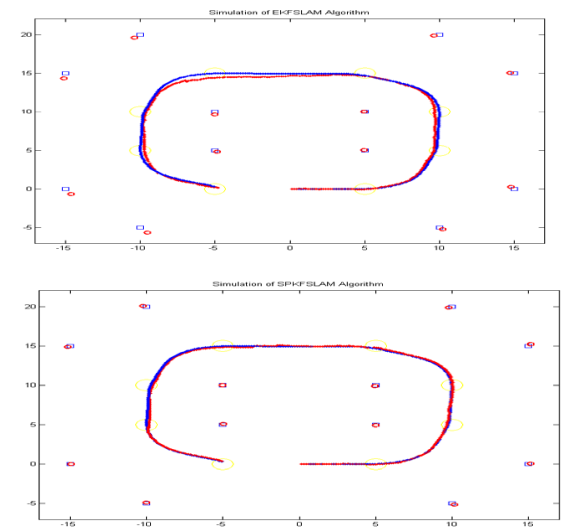

Fig.4. Comparing Robot position with EKF and SPKF

\section{Conclusion}

In this paper, we investigated the EKF and SPKF frameworks to improve the estimation accuracy in simultaneous localization and mapping. All of simulation results consistently verified better performance and more accurate states estimate of the SPKF than EKF approaches. After Comparing the Errors and the Position vector of the Robot with two different optimizations, SPKF had significant better result than EKF.

\section{References}

1. W.H. Durrant and T. Bailey, Simultaneous localization and mapping: Part I. IEEE Robotics and Automation Magazine, 2006, 13(2): pp. 99-110.

2. T. Bailey and W.H. Durrant, Simultaneous localization and mapping: Part II. IEEE Robotics and Automation Magazine, 13(3): 2006, pp. 108-117.

3. H. Durrant-Whyte and T. Bailey, "Simultaneous localization and mapping: Part 1", IEEE Trans. on Robotics and Automation, 13(2): 2006, pp. 99-108.

4. T. Bailey and H. Durrant-Whyte, "Simultaneous localization and mapping:Part II", IEEE Trans. on Robotics and Automation, 13(3): 2006, pp. 108-117

5. Y. Bar-Shalom, X. Rong Li, and T. Kirubarajan, Estimation with Applications to Tracking and Navigation. Chapter 10, Wiley InterScience, 2001, pp 372-380

6. S. Julier and J. K. Uhlmann, "A counter example to the theory of simultaneous localization and map building", in Proc. IEEE Int. Conf. Robot. Autom., 2001, pp. 4238-4243.

7. S. Huang and G. Dissanayake, "Convergence and consistency analysis for extended Kalman filter based SLAM", IEEE Transactions on Robotics and Automation, 23(5): ICRA02, Orlando, FL, USA, 2006, pp. 1036-1049.

8. J. Andrade-Cetto, T. Vidal-Calleja, and A. Sanfeliu. "Unscented transformation of vehicle states in SLAM." In Proc. of the 2005 IEEE Int. Conf. on Robotics and Automation, Barcelona, Spain, April 2005, pp. 324-329,

9. R. Martinez-Cantin and J. A. Castellanos, "Unscented SLAM for largescale outdoor environments." In Proc. of the 2005 IEEE Int. Conf. on Intelligent Robots and Systems, Aug. 2005, pp. 3427-3432.

10. Won-Seok Choi, Jeong-Gwan Kang and Se-Young Oh, Member, IEEE "Measurement Noise Estimator Assisted Extended Kalman Filter for SLAM Problem", 2009 IEEE/RSJ International Conference on Intelligent Robots and Systems, St. Louis, MO., United States, 2009, pp 2077-2082.

11. M. Yaghobi, M. Jadaliha, J. Zolghadr and M. Norouzi, Adaptive line extraction algorithm for SLAM application, Robotics and Biomimetics, Bangkok, Thailand, ROBIO 2008, pp 1850-1855.

12. Javad Zolghadr, Yuanli Cai, Locating a two-wheeled robot using extended kalman filter,, Technical Gazette 22, 6, 2015, pp. 1481-1488 